\title{
Dimensionamento econômico otimizado de redes de distribuição de água considerando custos de manutenção
}

\author{
Optimum economic sizing of water distribution \\ network considering maintenance cost
}

Marcos Rodrigues Pinnto', Marco Aurélio Holanda de Castro²,
João Marcelo Costa Barbosa ${ }^{3}$, José Valmir Farias Maia Júnior

\begin{abstract}
RESUMO
O dimensionamento econômico ótimo de redes de distribuição de água tem sido objeto de estudo de diversos pesquisadores e projetistas, e representa uma relevante parte nas ações de saneamento básico. No entanto, até então, abordase somente o custo de implantação, considerando que o custo dos condutos é um dos elementos de maior peso no projeto. Assim, o problema resume-se a dimensionar os condutos tendo como objetivo atingir uma rede de menor custo e que satisfaça os prérequisitos hidráulicos mínimos de dimensionamento. Isso conduz necessariamente à escolha de uma rede cuja combinação de condutos de menor diâmetro possível e que também seja capaz de satisfazer as restrições hidráulicas. Nossa abordagem, inédita até agora no Brasil, considera, adicionalmente, o custo de manutenção ao longo da vida útil da rede, tendo em vista que, se por um lado os condutos de maior diâmetro são mais caros, por outro lado, sofrem menos os efeitos da rugosidade eapresentam uma frequência menor de manutenção que os condutos de menor diâmetro, levando, assim, a uma rede que, ao longo da vida útil do projeto, apresenta um custo menor. Assim, obtém-se um equilibrio entre o custo de implantação e o custo de manutenção da rede.
\end{abstract}

Palavras-chave:saneamento básico; hidráulicacomputacional; Hidroinformática.

\begin{abstract}
The optimum economic sizing of a water distribution network has been focus of study for many researches and projectors, and represent a relevant part of sanitation programs. But, by now, the known works approaches only the implantation cost, considering that the pipes have biggest heavy in the project. So, the problem is reduced to sizing pipes which objective is reach a cheaper network and satisfying the minimum hydraulic prerequisites. This leads to the choice of a network with pipes of minor diameters since this choice satisfies the hydraulics restrictions. New in Brazil by now, our approach consider too the maintenance cost throughout life span of network. It because, if in one way the larger diameters are more expensive, in an other, it are more resistant to break and to rugosity effects. This leads to a network of minimum maintenance cost throughout its life span. Then, we reach the equilibrium between the implantation cost and the maintenance cost.
\end{abstract}

Keywords: sanitation; computational hydraulics; hydroinformatic.

\section{INTRODUÇÃO}

Uma rede de distribuição de água (RDA) pode ser vista como um grafo direcionado, no qual trechos e nós são representados por arestas e vértices, respectivamente (DIAS \& GOMES, 2007). Tecnicamente, uma RDA é um conjunto de condutos, junções e outros equipamentos específicos para viabilizar o transporte de água entre uma fonte (usualmente, um reservatório) e os pontos de consumo (em geral, as residências, comércio e indústrias).
Há diversas preocupações ligadas a uma RDA, desde o seu projeto e durante toda a sua vida útil. O atendimento da demanda (ALENCARNETO \& CASTRO, 2004; SHERALI \& SMITH, 1997), a minimização das perdas por vazamento (GONZÁLEZ-GÓMEZ et al., 2012), a ruptura de canos (NEELAKANTAN; SURIBABU; LINGIREDDY, 2008), o aumento de sua rugosidade (MOHAMED \& ABOZEID, 2011), a qualidade da água distribuída (BOIX et al., 2011; FERNANDES, 2004), e

'Doutor em Engenharia Civil pela Universidade Federal do Ceará (UFC) - Fortaleza (CE), Brasil.

${ }^{2} \mathrm{PhD}$ em Civil Engineering pela Drexel University (DU) - Philadelphia, Estados Unidos.

${ }^{3}$ Doutor em Engenharia Civil pela UFC - Fortaleza (CE), Brasil.

Mestre em Engenharia Civil pela UFC - Limoeiro (CE), Brasil.

Endereço para correspondência: Marcos Rodrigues Pinnto - Avenida Washington Soares, 1321 - 60811-341 - Fortaleza (CE), Brasil - mrp@unifor.br

Recebido: 10/09/14 - Aceito: 24/05/16 - Reg. ABES: 140349 
vulnerabilidade (PINTO et al., 2011) são alguns dos itens que requisitam atenção especial de projetistas e gestores.

A rugosidade, por exemplo, cresce dramaticamente com a idade da RDA e o diâmetro interno dos canos pode se reduzir significativamente devido a corrosão, deposição e acumulação de resíduos, resultando na diminuição drástica das capacidades hidráulicas do cano afetado (MOHAMED \& ABOZEID, 2011).

A implementação de uma RDA é reconhecidamente bastante onerosa, sendo então necessário o emprego de técnicas de otimização visando ao custo mínimo de implantação da mesma (SHERALI \& SMITH, 1997; ALENCAR-NETO \& CASTRO, 2004; CASTRO \& COSTA, 2004; DIAS \& GOMES, 2007; MAIA-JUNIOR, 2013). Contudo, no projeto de uma RDA surgem naturalmente mais de um objetivo, em geral, conflitantes, como éo caso, por exemplo, da minimização do custo de implantação versus o custo de manutenção da rede (NEELAKANTAN; SURIBABU; LINGIREDDY, 2008), da maximização da eficiência (RAAD; SINSKE; VUUREN, 2010).

Uma das dificuldades no processo de otimização de uma RDA é a disponibilidade de um número limitado de diâmetros no mercado. A combinação de canos de diversos diâmetros para compor a rede deve atender a restrições hidráulicas para que a mesma seja funcional. Canos com diâmetros maiores tendem a ser mais caros, sendo, portanto, preteridos na implantação. No entanto, canos de diâmetros menores tendem a quebrar com mais frequência e sofrer mais facilmente com o processo de aumento da rugosidade ao longo da vida útil da rede. Isso sugere que o projeto de uma RDA deve buscar balancear o custo de implantação com o custo de manutenção da rede, a fim de não perder toda a economia feita na implantação com uma manutenção mais frequente e mais cara.

Neelakantan, Suribabu e Lingireddy (2008) consideraram o caso biobjetivo custo mínimo de implantação e custo de manutenção comparando os resultados obtidos por um Algoritmo Genético (GA) sem a troca de canos com os resultados considerando-a. Boix et al. (2011) usaram três objetivos para o seu problema de otimização de uma RDA: o caudal de água doce na entrada da rede, a taxa de fluxo de água de entrada de unidades de regeneração, e o número de interligações de rede por meio de uma estratégia lexicográfica, na qual um procedimento de programação não linear mista (MINLP) é usado em cada passo.

Se por um lado os Algoritmos Evolucionários (AE) são ferramentas robustas e flexíveis, por outro são reconhecidamente custosas computacionalmente. Assim, optou-se por um algoritmo multipopulação e multiobjetivo. A estratégia de usar populações múltiplas é baseada no conceito de ilhas de injeção Multiscale Island Injection Genetic Algorithm (MIIGA), descrito por Sinha e Minsker, (2007). A abordagem multiobjetiva tem como base o conceito de Nicho de Pareto do Niched Pareto Genetic Algorithm (NPGA) descrito por Horn, Nafpliotis e Goldberg (1994).

Assim, empregou-se um AE multiobjetivo e multipopulação para obter a rede mais econômica, considerando o custo de implantação e simultaneamente o custo de manutenção.

\section{METODOLOGIA}

O algoritmo MINPGA foi aplicado ao problema de otimização de RDA, considerando-se dois objetivos: a minimização do custo de implantação e a minimização do custo de manutenção. No esquema de otimização foi utilizada a biblioteca OOTEN, uma codificação de diâmetros reais para inteiros consecutivos em um anel comutativo.

\section{Funcionamento do Algoritmo Multiscale Island Injection Genetic Algorithm}

O MINPGA utiliza a técnica de nichos de Pareto em conjunto com a técnica de múltiplas ilhas. Os nichos de Pareto são avaliados a fim de se resolver situações de conflito entre duas soluções para decidir qual delas será considerada melhor. Essa técnica consiste na contagem de soluções dentro dos raios centrados nas soluções conflitantes e de tamanho igual à metade da distância linear entre as duas soluções em questão. Da maneira como se definiu o raio, por exemplo, variável em vez de fixo, torna impossível a situação atípica de se ter as soluções conflitantes, incluindo-se mutuamente em seus nichos. Outra situação atípica evitada é a de que uma mesma solução faça parte de ambos os nichos das soluções em impasse. A solução que tiver o menor número de soluções dentro do seu raio de nicho é considerada vencedora.

O MINPGA inicializa três ilhas, cada uma com uma população inicial gerada de forma randômica. O fitness dos indivíduos é calculado de acordo com duas funções objetivo, que servirão de base para a ordenação dos mesmos. Cada indivíduo nessas populações está sujeitos às operações de cruzamento e de mutação. Tanto o cruzamento como a mutação são do tipo aritmética. As ilhas utilizadas melhoram a distribuição espacial da população inicial, além da diversidade.

Cada uma das ilhas executa operações de ordenamento, cruzamento, mutação. Uma das ilhas é considerada como principal e as outras duas como auxiliares. Há uma troca circular de indivíduos entre as ilhas. Após o ordenamento em cada ilha, a ilha 1 recebe uma cópia do indivíduo melhor colocado da ilha 3, que por sua vez recebe o indivíduo melhor colocado da ilha 2, a qual recebe da ilha 1 uma cópia do indivíduo melhor classificado naquela. Cada ilha, simultaneamente ao recebimento de um individuo de outra ilha, elimina o pior classificado. O processo se repete até que as populações tenham trocado o número completo de seus indivíduos menos dois (devido a operação de cruzamento, que necessita de pelo menos dois elementos).

O cruzamento ocorre entre dois indivíduos, um fixado e outro selecionado aleatoriamente. Um diâmetro em uma dada posição é escolhido e cruzado com outro diâmetro na posição correspondente do outro indivíduo. Um novo é gerado, mas somente é preservado se tiver fitness melhor do que os dois geradores.

Na mutação, escolhe-se uma posição aleatoriamente e muda-se o diâmetro de forma randômica (a mutação tem de ser uma mudança drástica). Cada novo indivíduo é avaliado e preservado somente quando tem fitness superior ao indivíduo que sofreu a mutação. 
O ordenamento dos indivíduos é feito considerando-se o conceito de dominância de Pareto, atribuindo-se posição 0 (zero) aos que não são dominados por qualquer solução. Na medida em que o torneio se desenvolve, a posição do indivíduo vai ganhando uma unidade cada vez que ele é confrontado com outro que o domina, forte ou fracamente. Assim, todos competem com todos para que o ordenamento seja completo. Os indivíduos com posição zero, i.e., não dominados por qualquer solução, formarão a frente de Pareto. A avaliação pelo nicho de Pareto somente é utilizada no caso de duas soluções estarem em conflito.

\section{Oriented Object Tookkit for EPANET}

A solução hidráulia das redes encontradas foi efetuada por meio da biblioteca para C++ do EPAnet, o TOOLKIT Oriented Object Tookkit for EPANET (OOTEN). Para isso, foi instalado o arquivo epanet2.dll e seu respectivo arquivo de cabeçalhos (.h). As funções utilizadas foram ENopen, ENsetlinkvalue, ENgetlinkvalue, ENsolve, e ENgetnodevalue.

A biblioteca OOTEN é baseada na toolkit para programadores do EPANET, e incorpora todas as funcionalidades dessa toolkit de forma orientada a objeto. Há ainda outras funcionalidades não presentes naquela toolkit. EPANET é escrito em ANSI-compatible C e OOTEN em ANSI C++. A OOTEN foi desenvolvida usando Borland $\mathrm{C}++$ Builder, versão 6.0, mas pode ser compilada em qualquer compilador ANSI C e C++ compatíveis. Nenhum componente visual, tais como janelas, estão referenciados no fonte do OOTEN. Como o código-fonte do EPANET, o do OOTEN também é de domínio público.

As funções da OOTEN são: abrir o arquivo INP; capturar o número de condutos na rede em estudo; capturar os comprimentos dos condutos; alterar os diâmetros da rede original; resolver hidraulicamente a rede; capturar as pressões nos nós para verificar a restrição hidráulica de que, exceto ao de um reservatório, a nenhum nó é permitido ter pressão menor que $10 \mathrm{mca}$.

ENopen abre o arquivo inp para utilização das funções de manipulação de parâmetros (get e set). ENsetlinkvalue permite mudar o valor dos diâmetros dos condutos (links) da rede. ENgetlinkvalue é utilizada para retornar o comprimento ou o diâmetro dos condutos. ENsolve resolve hidraulicamente a rede. ENgetnodevalue retorna o valor de parâmetros relacionados aos nós, neste caso, o valor da pressão em cada nó.

Para detalhes sobre a instalação e a utilização da biblioteca OOTEN, é possível consultar o endereço eletrônico http://epanet.de/developer/ ooten.html\#ooten.

\section{Codificação dos Diâmetros da Rede}

$\mathrm{O}$ diâmetro de um conduto é uma grandeza contínua. No entanto, os diâmetros comercializados têm medidas padronizadas que os colocam na condição de grandeza discreta. Por esse motivo, foi criada uma associação entre o conjunto dos nove diâmetros comercializados e o anel algébrico $Z_{9}$ com a operação soma.

Utilizando o anel em vez do conjunto de diâmetros reais, evita-se o inconveniente de os diâmetros não serem consecutivos, i.e., de haver infinitos números reais entre um diâmetro e outro. No anel, os elementos são consecutivos, possibilitando que dois princípios sejam seguidos:

1. a operação de cruzamento entre indivíduos de uma mesma espécie deve sempre gerar indivíduos da mesma espécie;

2. a operação de mutação não deve gerar indivíduos de espécie diferente da do indivíduo que sofreu a mutação.

Os dois princípios acima fazem sentido do ponto de vista da teoria sobre $\mathrm{AE}$, uma vez que a mudança sofrida pelos indivíduos diz respeito à combinação de seus genes e não a uma mudança no gene em si. Isto é, a combinação genética muda, o gene não.

Os diâmetros (internos) comerciais são associados aos elementos do anel algébrico $\mathrm{Z}_{9}$ de como é mostrado na Tabela 1 .

A vantagem de se utilizar o anel em vez dos diâmetros reais pode ser ilustrada com um exemplo: a soma do segundo com o nono diâmetro real é um número para o qual não há diâmetro previsto, isto é, 575.6; enquanto a soma dos respectivos elementos do anel, 1 e 8 , resulta em 0 , ou seja, o primeiro elemento do anel, ou um diâmetro previsto. Essa característica garante que os princípios (1) e (2), acima, estarão sendo seguidos.

A operação soma no anel algébrico $Z_{9}$ é dada no Quadro 1. A operação produto pode ser vista no Quadro 2.

Obeserva-se que o resultado da operação produto definida no Quadro 3 aplicada a dois elementos quaisquer do anel, sempre é um elemento do anel. Assim como na operação soma, a operação produto é feita entre os elementos da primeira coluna e os elementos da primeira linha. Garcia e Lequain (2005) apresentam mais detalhes sobre os anéis.

\section{Funções Objetivo}

Para a implantação de um projeto de RDA, o processo de otimização que vise ao custo mínimo resultará na escolha de uma rede que tenha os condutos de menor diâmetro (menor preço). Porém, os condutos de menor diâmetro têm taxas de quebra por quilômetro por ano maiores do que os de maior diâmetro (maior preço), impactando no custo de manutenção ao longo da vida útil da rede.

Tabela 1 - Codificuação de Diâmetros.

\begin{tabular}{|l|c|c|c|c|c|c|c|c|c}
\hline Diâmetro $(\mathrm{mm})$ & 54,6 & 77,2 & 100,0 & 156,4 & 202,2 & 252,0 & 299,8 & 394,6 & 498,4 \\
\hline Elemento de $Z_{9}$ & 0 & 1 & 2 & 3 & 4 & 5 & 6 & 7 & 8 \\
\hline
\end{tabular}

Fonte: autor. 
Logo, minimizar o custo de implantação e minimizar o custo de manutenção de uma RDA são objetivos conflitantes, sendo necessário definir uma função objetivo para cada custo, para que o AE avalie cada solução e efetue o torneio entre as mesmas.

O custo de implantação é calculado aplicando-se a Equação 1:

$C I=\sum_{i=1}^{n} \mathrm{C}_{d i} L_{i}$

Em que:

$C I$ = Custo de Implantação do projeto de RDA (R\$);

$n=$ Número de trechos presentes na RDA;

$C_{d}=$ Custo associado ao diâmetro no trecho i (R\$);

$L_{i}=$ Comprimento do i-ésimo trecho $(\mathrm{m})$.

O custo de manutenção (CM) é calculado por meio da Equação 2:
$C M=\sum_{j=1}^{r}\left(\sum_{k=1}^{t} p_{r} I_{(k)} l_{(k)}\right)$

Em que:

$C M=$ Custo de Manutenção do projeto de RDA (R\$);

$r=$ Número de anos de vida útil da rede considerada;

$t=$ Número de trechos presentes na rede;

$p_{r}=$ Taxa de quebra por período (número de quebra $/ \mathrm{km}$-ano);

$I_{(k)}=$ Custo de implantação por medida de comprimento do trecho $\mathrm{k}$ com diâmetro $\mathrm{d}_{(\mathrm{k})}(\mathrm{R} \$)$;

$l_{(k)}=$ Comprimento do k-ésimo trecho $(\mathrm{m})$.

Desde que a taxa de quebra anual por quilometro para condutos de uma RDA constitui-se uma estudo à parte e não se constituem objetivo do presente trabalho. Portanto, as taxas de quebra utilizadas na função objetivo Custo de Manutenção (CM) foram adaptadas da

Quadro 1 - Definição da Operação Soma (+) no Anel.

\begin{tabular}{|l|l|l|l|l|l|l|l|l|l|}
\hline$(+)$ & 0 & 1 & 2 & 3 & 4 & 5 & 6 & 7 & 7 \\
\hline 0 & 0 & 1 & 2 & 3 & 4 & 5 & 6 & 0 \\
\hline 1 & 1 & 2 & 3 & 4 & 5 & 6 & 7 & 0 \\
\hline 2 & 2 & 3 & 4 & 5 & 6 & 7 & 8 & 1 \\
\hline 3 & 3 & 4 & 5 & 6 & 7 & 8 & 0 & 2 \\
\hline 4 & 4 & 5 & 6 & 7 & 8 & 0 & 1 & 3 \\
\hline 5 & 5 & 6 & 7 & 8 & 0 & 1 & 2 & 3 \\
\hline 6 & 6 & 7 & 8 & 0 & 1 & 2 & 3 & 4 \\
\hline 7 & 7 & 8 & 0 & 1 & 2 & 3 & 4 & 5 \\
\hline 8 & 8 & 0 & 1 & 2 & 3 & 4 & 5 & 6 \\
\hline
\end{tabular}

Fonte: autor.

Quadro 2 - Definição da Operação Produto $(X)$ no Anel

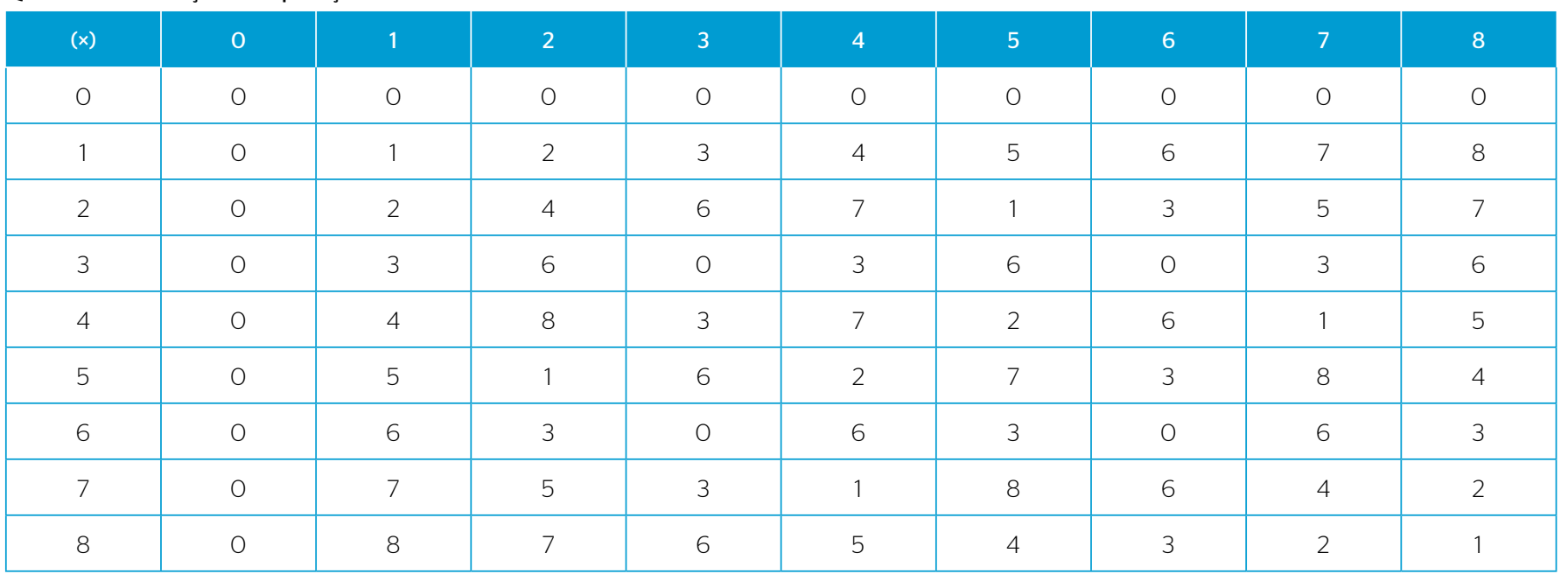


literatura. Os números de quebras por ano por quilometro no início do processo atribuídas a cada conduto de acordo com seu diâmetro estão dispostas na Tabela 2.

\section{Optimization Network by Evolutionary Algorithm}

O esquema de otimização se inicia com a abertura do arquivo contendo informações sobre a rede, como o número de trechos e o comprimento destes. Em seguida o ciclo do AE se inicia, gerando as populações aleatórias e não especialista com diâmetros codificados nas três ilhas consideradas. As redes então sofrem as operações usuais de um AE, gerando novas populações e repetindo o ciclo até que o critério de parada seja atingido. A frente de Pareto será formada com a população da ilha principal, sendo que as redes que compõem a frente devem ainda obecer a restrição hidráulica da pressão mínima em cada nó.

Quadro 3 - Funções analíticas para testes.

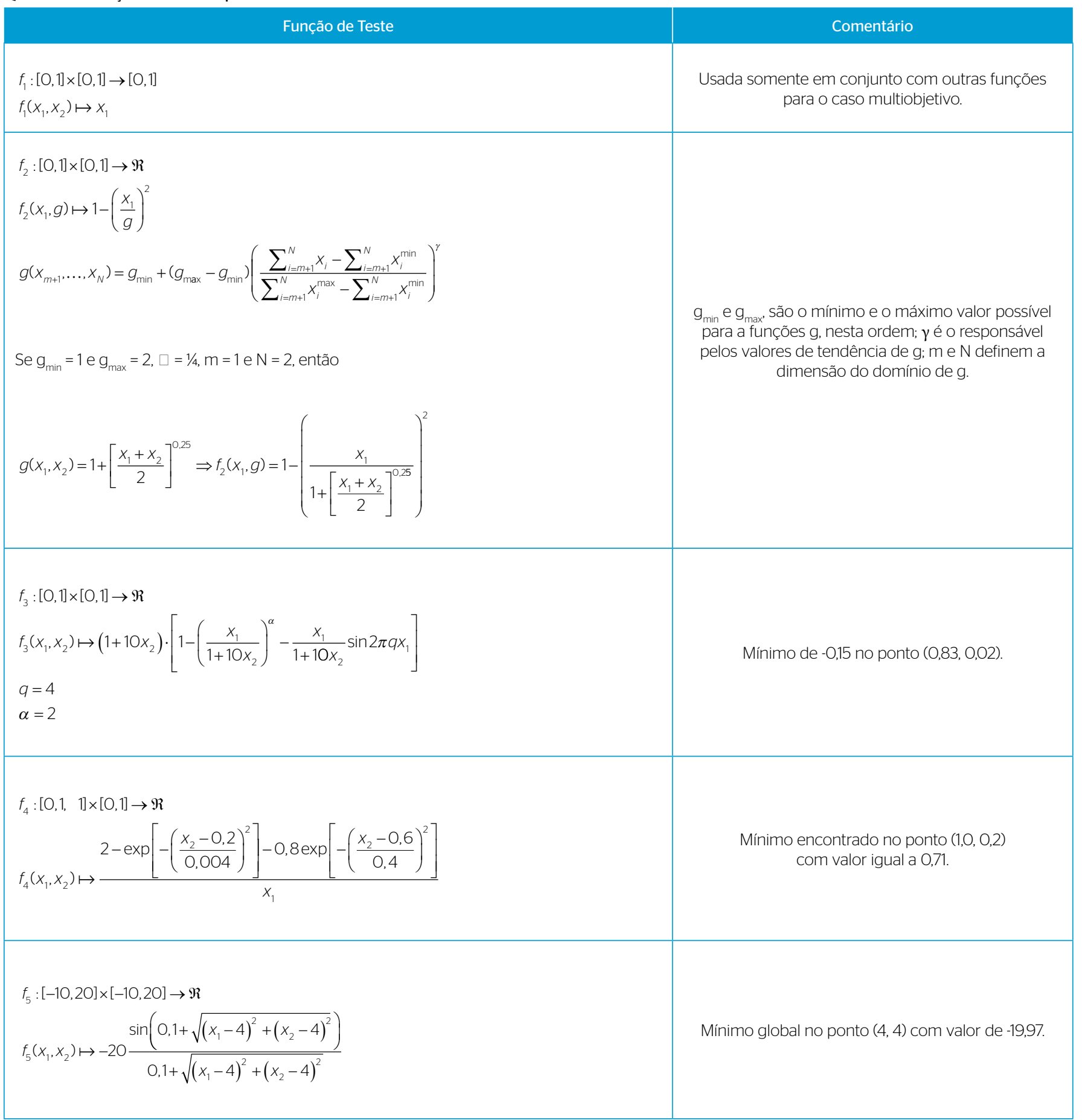




\section{Testes com Funções Analíticas}

O MINPGA foi submetido a testes com funções analíticas para verificação de sua acurária, precisão, flexibilidade e robustez. Essas funções também serviram para efetuar a análise de sensitividade do algoritmo e estabelecer os parâmetros que melhor se ajustariam ao mesmo. O Quadro 3 lista as funções com os respectivos comentários. Os parâmetros para MINPGA que obtiveram melhor resultados nos testes com duas funções objetivo estão listados no Quadro 4.

Testes utilizando as funções objetivos 1 e 2 geraram como resultado a frente de Pareto apresentada na Figura 1.

A Figura 2 mostra a formação da frente de Pareto para as funções objetivo 1 e 4 . A solução em destaque domina todas as outras, sendo portanto a melhor solução para o problema de minimização bi-objetivo ao considerar-se as funções objetivos citadas. O espaço visível entre as soluções dominadas significa que a solução destacada foi encontrada em uma geração anterior às últimas gerações, que foram descartadas.

Inicialmente pensou-se em impor tal restrição hidráulica desde o início do ciclo do $\mathrm{AE}$, o que aumentaria exponencialmente o tempo computacional, ou seja, o número de operações realizadas pelo programa computacional. Posteriormente, optou-se por fazer a verificação apenas com as redes que comporiam a frente de Pareto, evitando o inconveniente anterior. Isto foi possível porque, em problemas biobjetivo, cada função atua implicitamente como uma função de penalização do caso dos problemas uniobjetivo. Isto é, em problemas uniobjetivo, quando certa restrição não é atendida, uma função de penalização é acionada para tornar inviável aquela solução. No caso dos objetivos conflitantes, CI e CM, cada um atua implicitamente como função de penalização do outro, gerando certo equilíbrio nas soluções obtidas, o que de fato é esperado pelo conceito de frente de Pareto.

Para verificar as pressões em cada nó são chamadas funções do EPANET. Elas configuram a rede original e resolvem-na hidraulicamente, mostrando em fim as pressões nos nós. As pressões são avaliadas em cada nó e as redes que não atenderem à condição de pressão mínima são descartadas.

O esquema Optimization Network by Evolutionary Algorithm (ONEBEAR) é ilustrado com a Figura 3.

Tabela 2 -Taxas de Quebra.

\begin{tabular}{l|c|c|c|c|c|c|c|c|c|}
\hline Diâmetro (mm) & 54,6 & 77,2 & 100,0 & 156,4 & 202,2 & 252,0 & 299,8 & 394,6 \\
\hline $\begin{array}{l}\text { Taxa de Quebra } \\
\text { (quebra/km-ano) }\end{array}$ & 0,25 & 0,22 & 0,19 & 0,16 & 0,13 & 0,10 & 0,07 & 0,04 & 0,01 \\
\hline
\end{tabular}

Fonte: adaptada de Neelacantan et al. (2008).

Quadro 4 - Valores adotados para os parâmetros do MINPGA.

\begin{tabular}{|l|c|c|c|}
\hline Número de Populações & 3,000 & Raio de nicho & $*$ \\
\hline Número de soluções iniciais por população & 80,000 & Tamanho do torneio de seleção & 2,000 \\
\hline Probabilidade de cruzamento & 0,900 & Probabilidade de mutação & 0,001 \\
\hline Tipo de cruzamento e mutação & Aritmético & Inicialização da população & Aleatória \\
\hline Trocas entre as ilhas por geração & 1,000 & Peso da solução & via Aptidão \\
\hline
\end{tabular}

*variando com a posição das soluções conflitantes

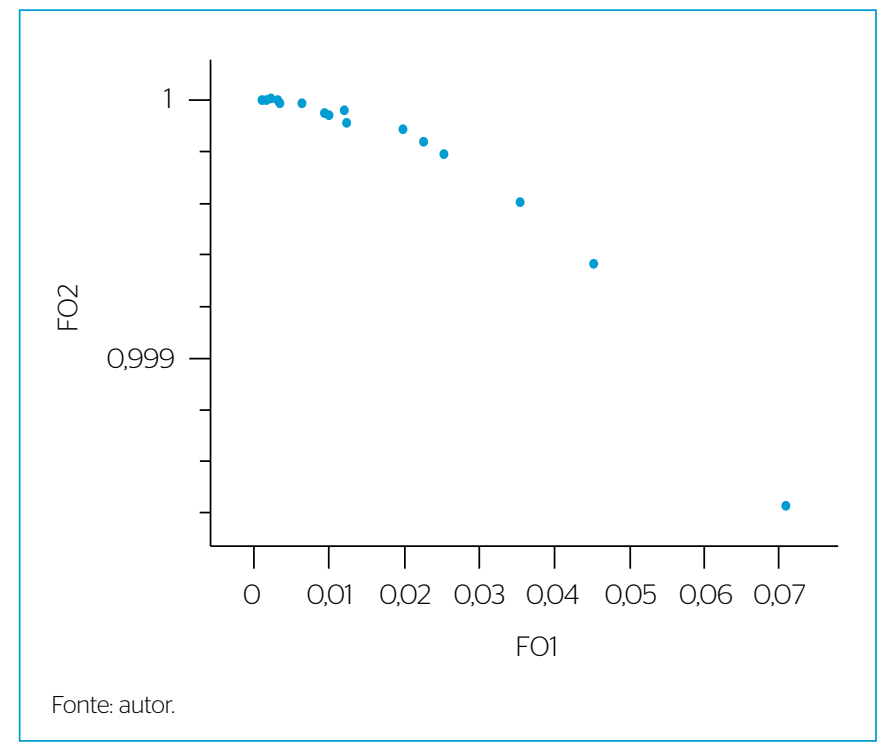

Figura 1 - Frente de Pareto para função objetivo 1 versus função objetivo 2.

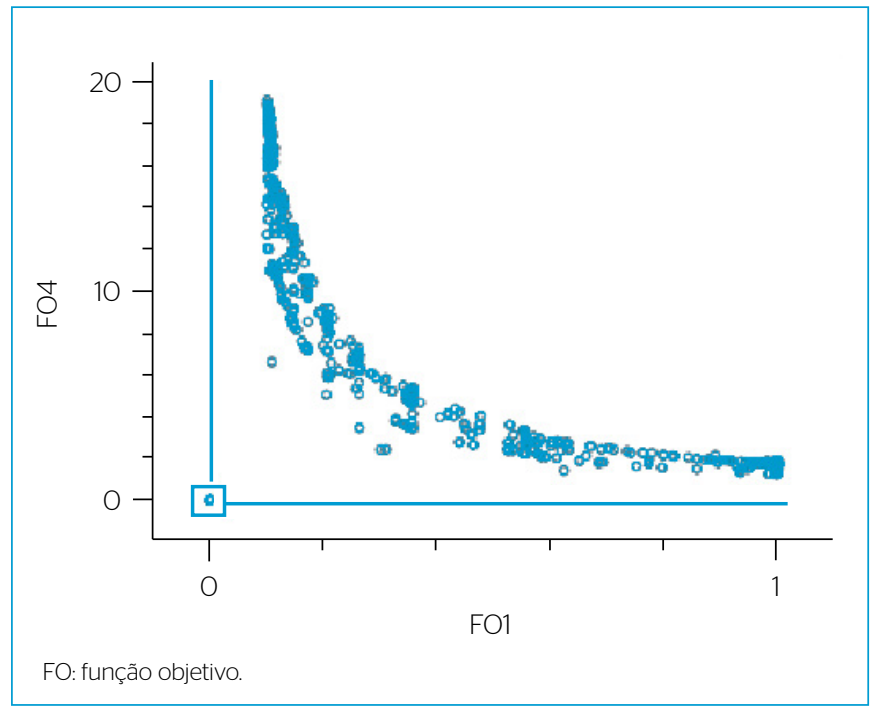

Figura 2 - Formação da frente de Pareto função objetivo 1 versus função objetivo 4. 


\section{RESULTADOS}

Dado que qualquer solução pertencente à frente de Pareto representa uma solução sub-ótima, e portanto adequada ao problema de otimização apresentado, apresentar-se-á a frente de Pareto obtida para a rede Açailândia submetida ao processo de otimização, escolhendo apenas cinco delas para listar seus respectivos diâmetros. A listagem de completa de todas as redes sub-ótimas encontradas, com seus diâmetros

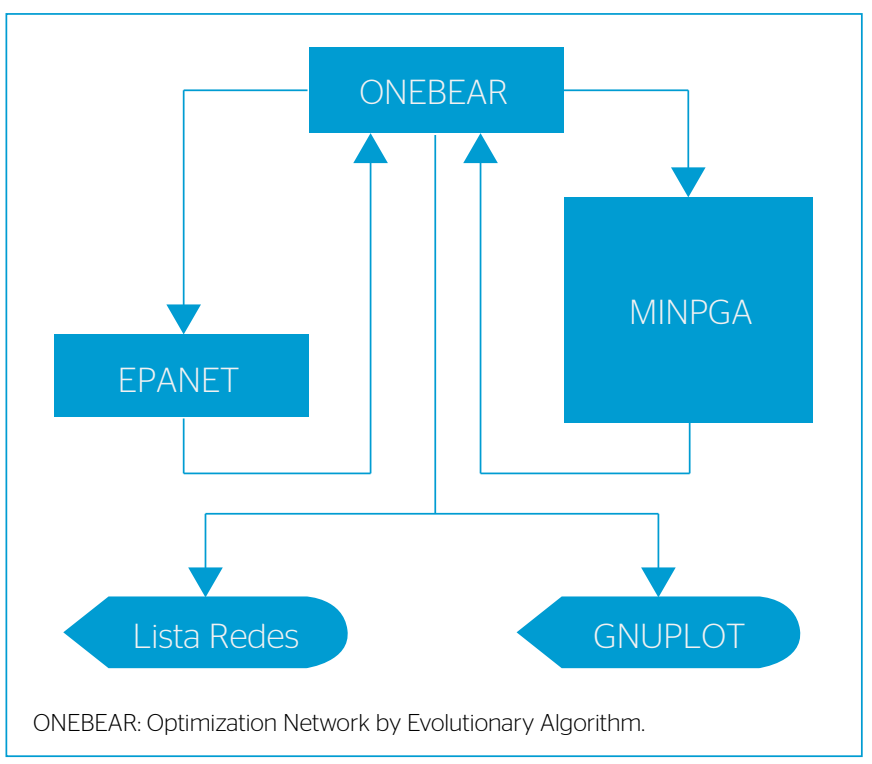

Figura 3 - Esquema Optimization Network By Evolutionary Algorithm. e trechos é feita em arquivo gerado pelo programa que implementa o esquema de otimização ONEBEAR.

\section{Rede Açailândia}

A rede Açailândia possui 666 trechos, 432 nós e 4 reservatórios. Trata-se de uma rede malhada cujo traçado pode ser visto na Figura 4.

A frente de Pareto gerada é apresentada na Figura 5.

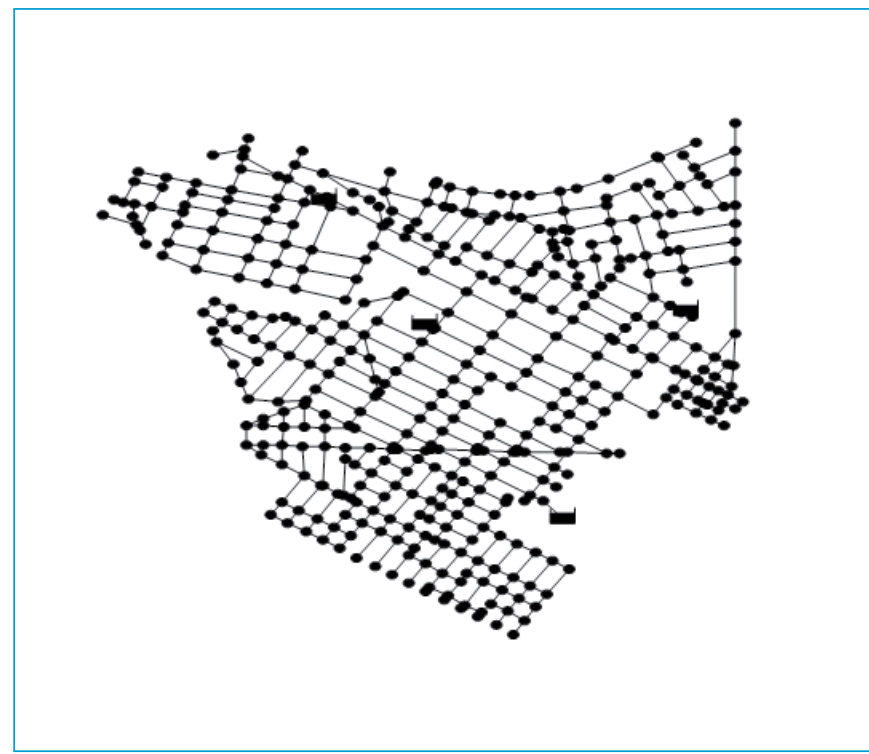

Figura 4 - Traçado da rede Açailândia.

\section{Frente de Pareto}

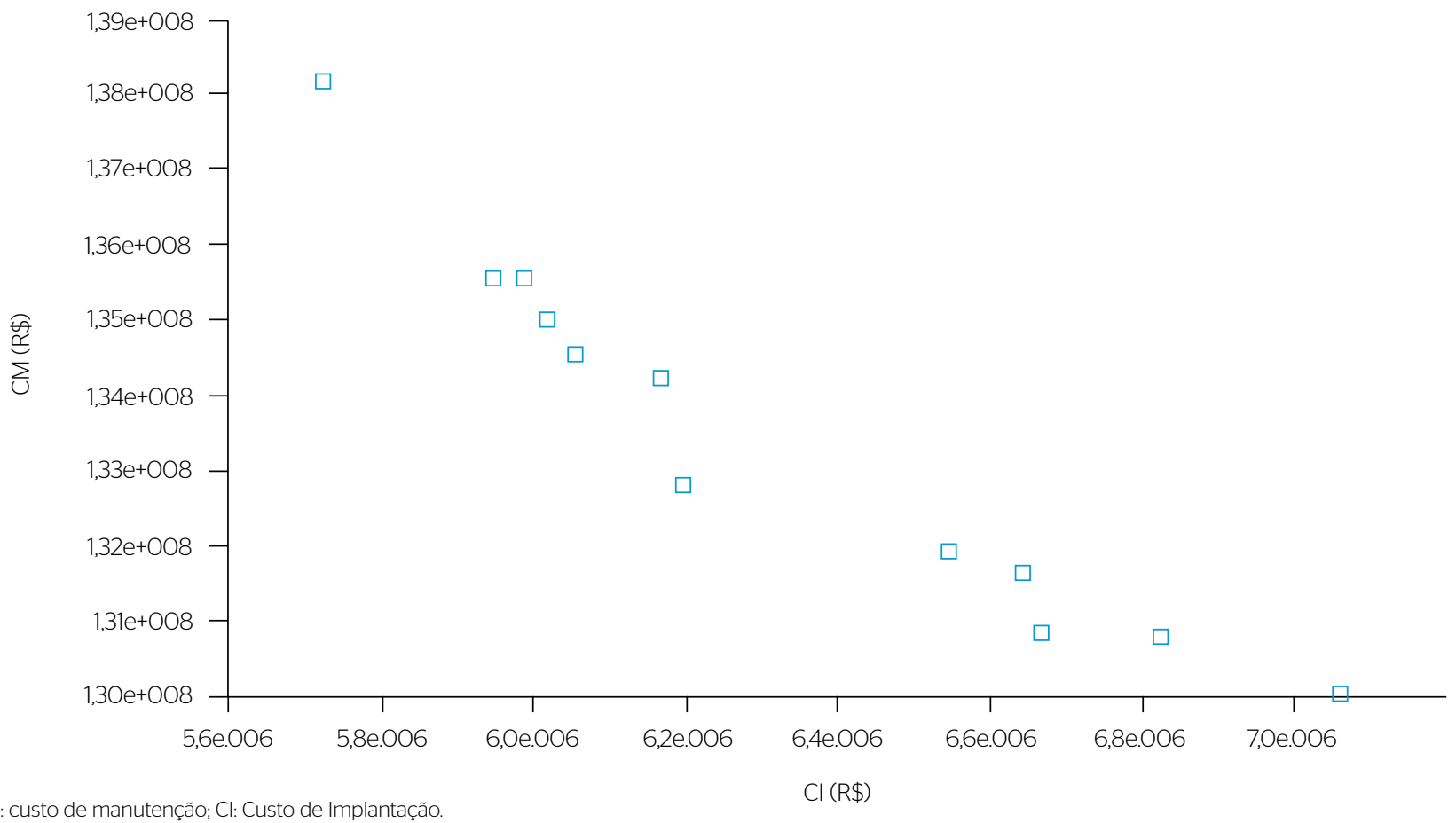

Figura 5 - Frente de Pareto custo de Implantação (R\$) versus custo de manutenção (R\$). 
De acordo com a Figura 5, com os parâmetros dados e considerando concomitantemente o custo de manutenção da rede ao longo de vinte anos, tem-se que a rede de menor custo de implantação custaria em torno de R \$ 5,7 milhões. Por outro lado, a rede com maior custo de implantação, nas mesmas condições, custaria cerca de $\mathrm{R} \$ 7,3$ milhões.

Ao analisar os custos de manutenção, que se baseia em taxas de quebra previamente definidas, observa-se que esse custo representa um valor que excederia o custo de implantação da rede completa em cada um dos vinte anos considerados. Isso pode passar a impressão de que as taxas de quebra estariam equivocadamente superestimadas. No entanto, essa comparação somente faz sentido considerando-se preços estáveis ao longo de vinte anos e tratando como nulo o custo de desinstalar toda a rede.

É importante lembrar ainda que outros custos, como o do inconveniente da suspensão do fornecimento, o do escavamento, o do possível desvio no trânsito, etc., não foram incluídos na metodologia apresentada. Contudo, isso pode ser feito por meio de ajustes nas funções objetivo.

Os custos associados cinco soluções dominantes que obedecem à restrição hidráulica sobre a pressão mínima são listados na Tabela 3.

\section{CONCLUSÕES}

As obras de saneamento são normalmente executadas por governos. A administração pública se norteia por princípios específicos para a sua gestão, entre eles, o princípio da eficiência, inserido pela Emenda Constitucional número 19, de 4 de junho de 1998. No entanto, essa eficiência, obter resultados eficazes e efetivos com o emprego da menor quantidade de recursos, cada vez mais deixa de ser uma variável de curto prazo, para se tornar uma variável de médio ou de longo prazo. Isto é, a busca pela economia de recursos deve ter reflexos positivos para além dos efeitos de curto prazo.

Portanto, oferecer uma metodologia capaz de minimizar custos de implantação de projetos de RDA e inserir o segundo objetivo de minimizar custos de manutenção ao longo da vida útil de uma rede constitui-se em uma ação relevante que pode contribuir diretamente para a eficiência da gestão pública e indiretamente para o desenvolvimento social do país, em especial, da região Nordeste.

Tabela 3 - Custos de cinco redes subótimas.

\begin{tabular}{c|c|c} 
Rede & CI (R\$) & CM (R\$) \\
\hline 1 & $5.984 .989,62$ & $135.517 .445,49$ \\
\hline 2 & $6.665 .092,64$ & $130.856 .237,12$ \\
\hline 3 & $6.641 .357,98$ & $131.657 .370,22$ \\
\hline 4 & $6.055 .569,03$ & $134.514 .530,00$ \\
\hline 5 & $6.130 .792,17$ & $134.478 .362,22$ \\
\hline
\end{tabular}

O esquema de otimização apresentado, ONEBEAR, que acoplou o motor hidráulico EPANET ao AE multiobjetivo e multipopulação MINPGA, atingiu seus objetivos de otimizar projetos de RDA visando ao custo mínimo de implantação e ao custo mínimo de manutenção ao longo de vinte anos de vida útil da rede.

Também é possível verificar que o ONEBEAR é flexível, pois pode ser aplicado a redes ramificadas, redes malhadas ou redes mistas. Isso é possível porque o MINPGA trabalha com apenas as informações sobre o fitness gerado por cada combinação de diâmetros, recusando as redes inviáveis apenas na formação da frente de Pareto. A flexibilidade do MINPGA é uma característica marcante dos algoritmos evolucionários em geral, e pode ser confirmada pelos testes com as funções objetivos analíticas.

Quanto à efetividade, pode-se dizer que o esquema alcançou as metas pretendidas de acoplar o EPANET e o MINPGA de forma a possibilitar o processo de otimização.

Quanto a eficácia, por meio do ONEBEAR é possível escolher a rede mais econômica quanto aos dois objetivos pretendidos e que atende à restrição hidráulica sobre a pressão mínimina em cada nó.

Quanto a eficiência, o esquema resolveu os problemas propostos em tempo computacional e com uso de memória aceitáveis em se tratando de AE.

O motor hidráulico EPANET vem sendo largamente utilizado nos problemas hidráulicos e de qualidade da água. Constitui-se em uma ferramenta de alta estabilidade e confiabilidade.

A biblioteca OOTEN está disponível no formato estático (LIB) e no formato dinâmico (DLL). Ela possibilitou o uso das funções do EPANET dentro do esquema de otimização, mostrou-se estável e confiável, não tendo sido verificado em nenhuma etapa do desenvolvimento qualquer erro oriundo dessa biblioteca.

As funções do EPANET foram determinantes para o sucesso do ONEBEAR, tendo sido utilizadas para abrir o arquivo com os parâmetros da rede, extrair determinados parâmetros, configurar os diâmetros e resolver hidraulicamente a rede.

A viabilidade da rede em termos da pressão mínima, por exemplo, foi obtida com uma dessas funções.

O fato de a biblioteca ser construída sob a técnica de orientação a objeto flexibiliza sua aplicação, torna o código altamente reutilizável, e facilita a modificação do código-fonte.

Como algoritmo híbrido, o MINPGA conserva algumas características dos algoritmos nos quais ele se baseia, o MIIGA e o NPGA.

A inicialização de três populações aumenta a diversidade e melhora as chances de convergência prematura do algoritmo. Além do mais, as trocas de indivíduos entre as populações oferece uma oportunidade de aprendizagem recíproca para cada uma das populações envolvidas no processo. $\mathrm{O}$ indivíduo pior adaptado de cada população cede lugar ao indivíduo mais adaptado da população vizinha. Esse elitismo composto contribui para uma convergência mais rápida, que pode ser obsevada mais claramente por meio de testes com funções analíticas. 
O nicho de Pareto foi utilizado para resolver aquelas situações em que duas redes ficam empatadas quanto a dominância, isto é, quando não se sabe quando qual das duas soluções é a dominante. Usar o raio de nicho flexível mostrou-se importante devido ao fato de contornar previamente duas possibilidades indesejáveis: os raios de nicho das duas soluções em conflito incluem uma mesma solução; os raios de nicho das duas soluções incluem uma a outra. Nas duas situações, o impasse seria mantido. Com o raio flexível, baseado na distância espacial entre as duas soluções, evita-se tanto um quanto o outro caso. Essa característica melhora a formação da frente de Pareto.

Os testes feitos com funções matemáticas mostraram ainda que o MINPGA é um algoritmo acurado e preciso. Acurado no sentido de que as soluções encontradas em dada geração se mostraram cada vez mais próximas das soluções obtidas na geração subsequente. Preciso pois foi verificada uma pequena magnitude da diferença entre a solução subótima dada pelo algoritmo e a solução ótima analítica do problema-teste, tendo em alguns casos convergido para a solução ótima.

Além disso, verificou-se que o algoritmo é robusto, ao ser aplicado a uma rede de 666 trechos. Considerando que se tem 9 diâmetros possíveis para cada um desses trechos, isso representa um problema de $6,73 \times 10^{19}$ combinações. Se cada uma dessas combinações pudesse ser testada em um nanossegundo, levar-se-ia mais de 2 mil anos para concluir o processo.

Isso mostra que a aplicação do MINPGA é adequada ao problema e oferece boas soluções em um tempo razoável.

\section{AGRADECIMENTOS}

O doutor Marcos Rodrigues Pinnto é pesquisador apoiado pela Fundação Edson Queiroz (Universidade de Fortaleza). O doutor João Marcelo Costa Barbosa é pesquisador apoiado pelo Conselho Nacional de Pesquisa e Desenvolvimento Científico e Tecnológico.

\section{REFERÊNCIAS}

ALENCAR-NETO, M.F. \& CASTRO, M.A.H. (2004) Projeto de redes de distribuição de água por algoritmo genético. In: IV Seminário Hispano-Brasileiro sobre Sistemas de Abastecimento Urbano de Água, João Pessoa (Brasil): SEREA.

BOIX, M., MONTRASTUC, L.; PIBOUULEAU L.; AZZARO-PANTEL, C.; DOMENECH, S. (2011) A Multiobjective optimization framework for multicontaminant industrial water network design. Journal of Environmental Management, v. 92, n. 7. p. 1802-1808.

CASTRO, M.A.H. de \& COSTA, C.T. (2004) Otimização de redes de distribuição de água usando a metodologia de Morgan e o EPANET. In: IV Seminário Hispano-Brasileiro sobre Sistemas de Abastecimento Urbano de Água, João Pessoa (Brasil): SEREA.

DIAS, C.H. \& GOMES, F.A.M. (2007) Otimização de redes de distribuição de água com estações de bombeamento. TEMA Tendências em Matemática Aplicada e Computacional, v. 8, n. 3 , p. $391-400$

FERNANDES, C.V.S. (2004) Modelagem da qualidade da água em redes de distribuição de água: a influência dos transientes hidráulicos, In: IV Seminário Hispano-Brasileiro sobre Sistemas de Abastecimento Urbano de Água, João Pessoa (Brasil): SEREA.

GARCIA, A. \& LEQUAIN, Y. (2005) Elementos de álgebra. 3. ed. Rio de Janeiro: IMPA. $326 \mathrm{p}$

GONZÁLEZ-GÓMEZ, F:; MARTÍNEZ-ESPIÑEIRA, R.; GARCÍAVALIÑAS, M.A.; GARCÍA-RUBIO, M.A. (2012) Explanatory factors of urban water leakage rates in Southern Spain. Utilities Policy, v. 22, n. 1, p. 22-30
HORN, J.; NAFPLIOTIS, N.; GOLDBERG, D.E. (1994) A Niched Pareto Genetic Algorithm For Multiobjective Optimization. In: Conference on Evolutionary Computation (ICEC '94), Piscataway, NJ: IEEE Service Center; p. 82-7.

MAIA-JUNIOR, J.V.F. (2012) Comparação de métodos de dimensionamento em redes de distribuição de água. 2012, 145 p. Dissertação (Mestado em Engenharia Civil - Recursos Hídricos) Universidade Federal do Ceará, Fortaleza.

MOHAMED, H.I. \& ABOZEID, G. (2011) Dynamic simulation of pressure head and chlorine concentration in the city of Asyut water supply network in abnormal operating conditions. Arabian Journal for Science and Engineering, v. 36, n. 2, p. 173-184,

NEELAKANTAN, T.R.; SURIBABU, C.R.; LINGIREDDY, S. (2008) Optimisation procedure for pipe-sizing with break-repair and replacement economics. Water SA. v. 34, n. 2, p. 217-224.

PINTO, J.; VARUM, H.; DUARTE, A.; AFONSO, L.; VARAJÃO, J.; BENTES, I. (2011) Risco de cenários de dano vulneráveis de redes de abastecimento de água. Territorium, v. 18, p. 271-278.

RAAD, D.; SINSKE, A.; VUUREN, J. (2010) Multiobjective optimization for water distribution system design using a hyperheuristic. Journal of Water Resources Planning and Management, v. 136, p. 592-596.

SHERALI, H.D. \& SMITH, E.P.A. (1997) Global optimization approach to a water distribution network design problem. Journal of Global Optimization, v. 11, n. 2, p. 107-132.

SINHA, E. \& MINSKER, B. (2007) Multiscale island injection genetic algorithm for ground water remediation. Advances in Water Resources, v. 30, n. 9, p. 1933-1942. 